\title{
LA MARIALOGÍA DE ELIZABETH JOHNSON EN DIÁLOGO CON EL CONTEXTO CHILENO
}

\author{
ELIZABETH JOHNSON'S MARIALOGY \\ IN DIALOGUE WITH THE CHILEAN CONTEXT
}

\author{
BLANCA BESA-BANDEIRA* \\ Mg. en Teología \\ Universidad Católica de Córdoba, Argentina \\ Unidad asociada al CONICET
}

Artículo recibido el I8 de noviembre de 202I; aceptado el 25 de diciembre de 2021 .

*blancabesa@gmail.com

https://orcid.org/oooo-0002-7969-0395

Cómo citar este artículo:

BESA BANDEIRA, B. "La marialogía de Elizabeth Johnson en diálogo con el contexto chileno" en Palabra y Razón. Revista de Filosofía, Teología y Ciencias de la Religión. № 20 DICIEMBRE 202I, pp. 54-73 https://doi.org/10.29035/pyr.20.54 


\title{
RESUMEN
}

La figura de la Virgen María sigue, al día de hoy, impactando en la vida de las mujeres. Con esta convicción este estudio ensaya una relectura de la obra marialógica de Elizabeth Johnson Verdadera hermana nuestra. Teología de María en la comunión de los santos, desde el contexto chileno. El foco del estudio está en la antropología feminista sobre la cual Johnson construye su teología de María analizando cuáles son las principales implicancias de una antropología de estas características, tanto para el tratado marialógico, como para las mujeres de nuestro país. Para esta segunda cuestión, se profundizará en el proceso de construcción de la identidad de las mujeres chilenas, relevando la importancia de considerar los contextos socioculturales a la hora de pensar una marialogía feminista. Desde la realidad chilena, la maternidad y la religiosidad popular aparecen como elementos que la reflexión sobre María no puede soslayar.

Palabras claves: marialogía feminista / antropología / mujeres chilenas / maternidad / religiosidad popular

\begin{abstract}
The figure of the Virgin Mary continues, to this day, impacting the lives of women. With this conviction, this study rehearses a rereading of Elizabeth Johnson's marialogical work, True our Sister. Theology of Mary in the communion of saints, from the Chilean context. The focus of the study is on feminist anthropology on which Johnson builds his theology of Mary by analyzing the main implications of an anthropology of these characteristics, both for the marialological treatise and for the women of our country. For this second question, the process of construction of the identity of Chilean women will be deepened, highlighting the importance of considering sociocultural contexts when thinking about a feminist marialogy. From the Chilean reality, motherhood and popular religiosity appear as elements that reflection on Mary cannot ignore.
\end{abstract}

Keywords: feminist marialogy / anthropology / chilean women / motherhood / popular religiosity 


\section{Introducción}

El libro Verdadera hermana nuestra. Teología de María en la comunión de los santos $^{\mathrm{I}}$ de Elizabeth Johnson puede considerarse como una de las obras de referencia de la marialogía ${ }^{2}$ feminista contemporánea ${ }^{3}$. La convicción de la autora norteamericana es que reconocer a María desde su realidad de mujer, impacta en la vida de las mujeres hoy, en sus relaciones y en el lugar que ellas ocupan en la Iglesia y en la sociedad. Compartir esta convicción perfila el objetivo de este artículo, que ensaya una relectura de la propuesta de Johnson desde el contexto chileno.

Uno de los grandes propósitos de Johnson es «distanciarse de toda simbolización recuperando la realidad concreta de María como mujer de Nazaret» ${ }^{4}$. Porque los símbolos no son éticamente neutros y, sin duda alguna, la figura de María ha formado parte de la comprensión de la imagen de la mujer en nuestra cultura occidental5. La simbología mariana ha tocado -y sigue tocando- aspectos fundamentales de la identidad de las mujeres: género, sexo, rol, imagen, conductas, valores asumidos ${ }^{6}$, es decir, cuestiones que tienen que ver con la cultura, con el modo cómo comprendemos la realidad, vivimos y nos relacionamos. Así, lo que Johnson pretende es «releer la figura de esta mujer desde la perspectiva de las mujeres, buscando lo que en María puede ayudarlas a tomar distancia de modelos que las inferiorizan ${ }^{7}$. Para esto distribuye la obra «en dos momentos, el primero de crítica deconstructiva y el segundo de carácter narrativo reconstructivo y propositivo» ${ }^{8}$. Es en el primer paso de deconstrucción en el cual se aborda, sobre todo, la cuestión del símbolo.

I E. JOHNSON - D. ROMERO (trad.). Verdadera hermana nuestra. Teología de María en la comunión de los santos. Herder: Barcelona, 2005.

2 Siguiendo a De Fiores (1988, p.1287), usaremos para este artículo el término marialogía. Principalmente porque este no deforma el nombre de María y, así, sirve como acto simbólico de la intención por respetar su identidad y realidad de mujer por sobre los constructos y estereotipos sobre ella.

3 Cf. Recensión de María Josefina Llach sobre la obra de E. JOHNSON. Truly Our Sister. A Theology of Mary in the Communion of Saints. Cf. V. AZCUY et al. (coord.), Diccionario de autoras en América Latina, el Caribe y Estados Unidos (Mujeres haciendo teologías I; San Pablo: Buenos Aires 2007); G. ROSOLINO, "María ¿'La mujer, ícono del misterio' o 'Verdadera hermana nuestra'?" en Revista Teología. 96 (2008), pp. 33I-352; V. AZCUY, "Hablar rectamente de María. Mapas y perspectivas de mariología feminista" en V. AZCUY et al. (ed.) Teología feminista a tres voces. Universidad Alberto Hurtado: Santiago de Chile, 2016, pp. 247-266.

4 G. ROSOLINO. “María...", p. 350.

5 Cf. E. VUOLA, "La virgen María como ideal femenino, su crítica feminista y nuevas interpretaciones" en Pasos. 45 (I993), p. 20.

6 M. NAVARRO, "Buscando desesperadamente a María. La recuperación feminista de la mariología" en Ephemerides Mariologicae. 45/4 (1995), pp. 457-482, p. 458.

7 M. LLACH. Truly Our Sister..., p. I4I.

8 G. ROSOLINO. “María...", p. 34I. 
La crítica apunta a la antropología androcéntrica que permite sostener interpretaciones idealizadas de la figura de María como ideal de mujer o rostro maternal de Dios, proponiendo, en cambio, una antropología igualitaria del compañerismo. Como segundo paso metodológico, Johnson propone una reconstrucción del símbolo mariano en términos de igualdad. Para esto, plantea una marialogía pneumatológicaeclesiológica, tomando como referente el símbolo de la comunión de los santos, un símbolo intrínsecamente inclusivo e igualitario.

Desde mi punto de vista, la teología de María de Johnson tiene dos núcleos centrales: su propuesta antropológica -sobre la cual la autora construye su planteo-, y su desarrollo pneumatológico-eclesial. Este artículo se propone abordar, en primer lugar, la cuestión antropológica, mostrando en qué medida este elemento es el que posibilita y abre a una comprensión renovada del tratado. Como segundo momento de la reflexión, se plantea cuáles pueden ser las resonancias de una marialogía feminista desde el contexto religioso-cultural chileno y en qué medida una teología feminista de María podría representar un aporte para la situación actual de las mujeres en Chile. Para este análisis, será necesario primero presentar algunos de los elementos que han estado en juego en la conformación de la cultura y la identidad de las mujeres chilenas. Porque, conocer la historia de cómo se fue conformando esta identidad, es lo que permitirá reconocer cuáles son los posibles aportes de una marialogía de estas características. Esta explicación se hará a partir de los aportes y estudios de la antropóloga chilena Sonia Montecino.

Ahora bien, aunque el punto de partida de la reflexión es el planteo de Johnson, este momento reflexivo pretende ir más allá de ella, señalando también algunos desafíos que la experiencia religiosa inculturada presenta a las marialogías feministas -particularmente a la de Johnson-. Así, hacia el final del artículo, se propondrán algunos caminos que, me parece, podrían ser fructíferos para una teología de María pensada y vivida desde este país.

\section{La antropología igualitaria del compañerismo como elemento fundante}

En la obra, el objeto de la crítica de Johnson son las mariologías patriarcales porque le parecen conducentes a injusticia al estar construidas sobre una antropología dualista. Ésta, propone un modelo binario que, con una concepción esencialista del género atribuyen de forma fija características y roles a mujeres y varones. La relación entre 
mujeres y varones se entiende por medio de la complementariedad, cuestión a la que Johnson apunta, pues permite mantener a las mujeres en una posición de subordinación. Porque por «su definición las mujeres son siempre relegadas al ámbito privado y pasivo»" ${ }^{9}$, por tanto, entendidas como el suplemento de los varones, a quienes corresponde la iniciativa y el ámbito público.

En contraposición a esta antropología, ella propone una que ponga el acento en la pluralidad de factores que definen a la persona humana, entendiendo, con ello, que el sexo no es el único factor determinante, sino uno más entre otros igualmente importantes, razón por la cual su postura podría llamarse: antropología multipolar ${ }^{\mathrm{I}}$. Lo prioritario es salir de la perspectiva binaria -masculino y femenino- o de reducir a varones y mujeres a un determinado ideal para abrirse a descubrir la diversidad de modos de ser humano. Así, lo multipolar refiere a las múltiples combinaciones de elementos humanos esenciales, que entran en la definición de una persona. De manera que el ser humano deja de estar definido en referencia a un solo núcleo -el sexo-, sino que son muchos los polos que influyen y marcan a una persona: raza, clase, familia, geografía, cultura, sociedad, etc. Por tanto, esta antropología rescata el hecho de que la existencia humana tiene un carácter multidimensional. Las posibilidades de combinación de los elementos constitutivos de la humanidad de cada persona son siempre distintas, pues, si cambiamos de modo significativo cualquiera de ellas, surge una persona diferente. «Concentrarse en la sexualidad, con la exclusión de otros elementos igualmente constitutivos, es el equivalente de usar un microscopio para este factor clave de la vida humana cuando lo que se necesita es un telescopio para captar las galaxias de la rica diferencia humana» ${ }^{\mathrm{II}}$.

La misma Johnson señala que esta antropología de la reciprocidad o mutualidad es propia de la teología feminista de la liberación, la cual está todavía en desarrollo. Una autora pionera en este planteo es L. M. Russell, con su concepto de partnership [compañerismo] como un modo nuevo de pensar las relaciones humanas en términos de igualdad, cooperación e inclusión ${ }^{12}$. Desde América Latina, encontramos a I. Gebara y M. C. Bingemer. En el capítulo I de su obra conjunta María,

9 E. A. JOHNSON, "La masculinidad de Cristo" en Concilium. 226 (1989), pp. 489-499, p. 49 I. Io Cf. E. A. JOHNSON. La que es. El misterio de Dios en el discurso teológico feminista. Herder: Barcelona, 2002, P. 208.

II E. A. JOHNSON. La masculinidad..., 493ss.

I2 Cf. L. RUSSELL. The Future of Partnership. The Westminster Press: Philadelphia, 1979. 
madre de Dios, madre de los pobres ${ }^{13}$, Gebara desarrolla una antropología humanocéntrica, unitaria, realista y pluridimensional para el desarrollo de una reflexión sobre María. Pilar Aquino recoge estos aportes y explica que, lo que las autoras plantean, es un movimiento de una antropología androcéntrica a una humanocéntrica (centrada en la humanidad). De una antropología dualista que opone espíritu-materia y distingue dos historias, una profana y otra sagrada, hacia una antropología unitaria, que afirma la profunda unidad del ser humano, material-espiritual a la vez y es parte de una sola historia humano-divina. De una antropología idealista, que pone la verdad de la humanidad más allá de la historia, hacia una antropología realista, que asume la realidad humana tal como se nos presenta en su contingencia, conflictividad, contradicción, así como en sus luchas y esperanzas. De una antropología unidimensional, donde las personas son una idea o una definición, hacia una antropología pluridimensional, donde las personas son rostros históricos que incluyen múltiples dimensiones, marcados por el espacio y el tiempo. En definitiva, una antropología que no suprime lo diferente, sino que, más bien, acepta el desafío y el misterio de nuestra extraordinaria diversidad ${ }^{14}$.

Johnson considera que el pensamiento feminista resiste a dos $\operatorname{cosas}^{15}$ : por un lado, el punto de vista de la polaridad sexual de la naturaleza humana, que necesariamente conduce a un modelo dominante/ subordinado; y, por otro, la reducción a una uniforme igualdad que ignora la diferencia. Así, el cambio que se propone en la antropología multipolar es del esquema de dos términos o de un término, a uno de término múltiple que, más que garantizar constantemente la identidad mediante la oposición o la uniformidad, permite la conexión en la diferencia. En este sentido, frente al sistema binario de la antropología dualista, la propuesta feminista de Johnson no es una antropología neutra o unisex que anule las diferencias. Al contrario, se trata de reconocer la diferencia existente entre varones y mujeres, pero mostrando que, a esta diferencia, no le pertenece un espectro determinado de características. Las múltiples características humanas y las capacidades personales no dependen del sexo biológico de la persona ni vienen predeterminadas por él, sino que éstas pueden darse indistintamente, tanto en varones como en mujeres.

I3 I. GEBARA - M. C. BINGEMER. María, madre de Dios, madre de los pobres. Paulinas: Madrid, 1987.

I4 Cf. M. P. AQUINO, "Presupuestos metodológicos de la teología desde la perspectiva de la mujer" en C. L. AJO - M. DE LA PAZ (comp.) Teología y Género. Selección de textos. Caminos: La Habana, 2002, pp. I52-154.

I5 Cf. E. A. JOHNSON. La masculinidad..., p. 492. 
Lo que Johnson plantea es que, más que fijarse en la diferencia, hay que apuntar a lo que tenemos en común como especie que es -nada más y nada menos- que la naturaleza humana. Este tiene que ser el punto de partida: lo mucho que tenemos en común, sin que esto signifique olvidarse de la diferencia y menos de la corporeidad, la cual, por lo demás, es un elemento muy importante para la teología feminista. Establecida una igualdad fundamental, es posible que se dé la solidaridad en la diferencia. Es decir, manteniendo nuestra identidad distinta, podemos vincularnos más allá de nuestro sexo, con muchos otros varones y mujeres. Por tanto, esta antropología tiene un carácter liberador. Pues, si la asociación de determinadas características a cada uno de los sexos ha sostenido la concepción estereotipada de roles sociales predefinidos y separados, con la consecuente subordinación de las mujeres, pensar la masculinidad y la feminidad en un contexto más holístico permite concebir su relación humana de modo más correcto. Porque, desde esta antropología renovada, no es posible establecer jerarquías entre los seres humanos y, por tanto, las relaciones son liberadas del paradigma dominación-subordinación para transitar hacia uno de la igualdad, amistad y reciprocidad.

\section{Comprensión renovada de la marialogía desde la antropología feminista}

Como ya se planteó, si bien se reconoce que la creatividad y novedad del pensamiento de Johnson radica en el hecho de insertar a María dentro la comunión de los santos a partir de la pneumatología, sostengo que su planteamiento se construye a partir de una antropología feminista que pone los medios para entender esa comunidad y al mismo ser humano -y por tanto también a María- de un modo determinado. De manera que esta antropología pone los medios para pensar la marialogía desde otro paradigma distinto del patriarcal o androcéntrico y de aquí que Johnson afirme la necesidad de, a la hora de repensar la persona de María «mantener operando nuestra antropología, desenmascarando definiciones de género de lo femenino que confinan a las mujeres a roles subordinados» ${ }^{16}$.

Una primera implicancia de esta comprensión antropológica para la teología de María tiene que ver directamente con el propósito de la obra de Johnson: comprender a María formando parte de la comunión de las santas y santos y, desde aquí, recordarla como un ser humano histórico

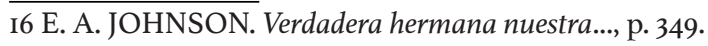


concreto que llevó adelante su vida en el Espíritu. Para Johnson, el hecho de ser una mujer agraciada -habitada por el Espíritu- es lo que pone a María en conexión con la comunidad de todos los santas y santos de Dios. Porque, dado que María «está poseída por el EspírituSofía y ante él responde, ella es una hermana para cuantos colaboran con el Espíritu en la lucha por la llegada del Reino de Dios» ${ }^{17}$. Apoyada en esta antropología, la teóloga norteamericana entiende la comunión de las santas y santos como una comunidad que, al moverse en un paradigma distinto al tradicional, posibilita imaginar un tipo diferente de comunidad. Una cuyas relaciones no se mueven en un plano de dominación-subordinación, sino que están marcadas por la reciprocidad y la amistad, en la cual la igualdad de cada uno de sus miembros no desdibuja la particularidad y diversidad de cada una(o). Esta es, entonces, una antropología que tiene efectos prácticos y críticos. Así, insertar a María dentro de esta comunidad, permite entenderla, no ya en un esquema de relaciones jerárquicas, sino como verdadera hermana nuestra y, como tal, miembro de la comunidad de las santas y santos de Dios.

De esto se desprende un segundo elemento que tiene que ver con el vínculo de solidaridad que podemos establecer con María cuando nos acercarnos a ella como mujer agraciada. Considerarla siendo parte ella misma de la gran comunidad de los agraciados, de las mujeres y varones habitados por el Espíritu: una multitud de hermanos y hermanas ${ }^{18}$. María, entonces, queda situada en medio y siendo parte de la comunidad de discípulas(os) que se dejan guiar por el Espíritu, para ser sus colaboradores en la construcción del Reino. Hay, entonces, entre María y el resto de la comunidad un vínculo profundo, mediado y posibilitado por el Espíritu de Dios, principio de unidad ${ }^{19}$. Así, María es parte de la comunidad intergeneracional de las y los creyentes (comunión de los santos) que han realizado su vida en apertura y relación con el Espíritu, lo que no anula su identidad, es decir, la misión única, específica e irrepetible que ha recibido de parte de Dios como madre del Salvador. Por tanto, los dones que María recibe, en orden a su misión, no la desvinculan de la comunidad de discipulado ni menos aún de la humanidad.

Esta manera de comprender al ser humano como único e irrepetible, pero, al mismo tiempo, en profunda relación con todas(os) quienes pertenecen a su raza, es lo que la antropología feminista expresa

I7 E. A. JOHNSON. Verdadera hermana nuestra..., p. 132.

I8 Cf. V. AZCUY, “Hablar rectamente de María ...", p. 256.

I9 CONCILIO ECUMÉNICO VATICANO II, Constitución Dogmática Lumen Gentium, sobre la Iglesia 4, 13. Roma, 1964. 
por medio del término ya descrito de solidaridad en la diferencia y que marca también el modo cómo Johnson comprende la comunión de los santos y en la cual sitúa su reflexión sobre María.

En este punto es interesante notar que la autora no restringe la fundamental igualdad entre mujeres y varones, y las relaciones de compañerismo que el Espíritu suscita, únicamente al plano eclesial, "porque el Espíritu no limita su bendición divina a un único grupo» ${ }^{20}$. De manera que la comunión de los santos no es un concepto que sólo alude a la comunidad eclesial. Al contrario, afirma que hay un vínculo entre todas las mujeres y varones, que han sido tocados por el fuego del amor divino y procuran vivir a Dios en sus vidas. Desde esta perspectiva, la comunión de los santos aparece como un concepto intrínsecamente inclusivo y muestra hasta dónde llega la convicción de Johnson de que nadie quede fuera y todo ser humano pueda ser reconocido igualmente como hermana(o). Un planteamiento que resulta ser no sólo feminista, sino también pluralista y multicultural, reconociendo la gran riqueza y diversidad de los seres humanos, buscando tender puentes que permitan el encuentro.

Por otra parte, esta antropología igualitaria tiene también implicancias para la cuestión del símbolo mariano. Desde la comprensión dualista de género, la interpretación de María resulta sexista, pues las concepciones de María derivan inevitablemente hacia vías que conducen a la idealización de lo femenino como contrapuesto de lo masculino. Y la idealización de la figura mariana «ha funcionado eficazmente para mantener a las mujeres en su lugar previamente asignado de subordinación a la autoridad patriarcal $»^{2 \mathrm{I}}$. De manera que, en un contexto androcéntrico, en vez de desafiar al sexismo, María se ha transformado en su ícono, legitimando estructuras sociales patriarcales. Ahora bien, una antropología distinta permite, por un lado, demoler esos supuestos sexistas y, por otro lado, abrirnos a una interpretación diferente de la figura mariana, libre de estereotipos de género, que no idealice ni sacralice virtudes conducentes a la sumisión de la mujer y que permita acercarse a su compleja identidad de una manera que dé mejor cuenta de sus distintos rasgos y facetas. Pero, una interpretación renovada de María supone primero una antropología que la fundamente, como se ejemplificará en el próximo apartado.

20 E. A. JOHNSON. Verdadera hermana nuestra..., p. 350.

2I E. A. JOHNSON. Verdadera hermana nuestra..., p. 25. 


\section{Aportes para una nueva comprensión de las mujeres chilenas}

Aunque en la obra se vea un claro esfuerzo por llevar adelante el planteamiento en solidaridad, sobre todo, con las mujeres más pobres, el contexto de Johnson es distinto al nuestro. El lugar desde el cual la autora lleva adelante su reflexión es una cultura postindustrial frecuentemente caracterizada como postmoderna, propia de una norteamericana blanca de clase media. Latinoamérica, en cambio, es un continente cuya realidad de pobreza hace a las mujeres tres veces pobres, debido a la opresión social, sexual y étnica que muchas de ellas sufren. Ya Aparecida habla de las mujeres sometidas a toda forma de exclusión y violencia: "entre ellas, las mujeres pobres, indígenas y afroamericanas han sufrido una doble marginación"22.

Como se señaló antes, resulta interesante para nuestra reflexión aproximarse a pensamiento de Sonia Montecino, quien, en diversos estudios, ofrece una comprensión del proceso de construcción de la identidad femenina en Chile ${ }^{23}$. A partir del análisis que ella plantea, haremos un intento por dilucidar cómo funcionaría una marialogía feminista en el contexto cultural chileno de sesgo machista -siguiendo a Montecino-, el cual tiende a idealizar a las mujeres como madres y, por tanto, prefiere la figura de María madre.

Para la antropóloga, las raíces de la conjunción en la cultura chilena entre una fuerte valoración de la madre -cuya imagen dominante se encuentra en el modelo mariano- por un lado, y el machismo, por otro, estarían en el mestizaje social y el sincretismo religioso que se gestó en la época de la conquista ${ }^{24}$. Es justamente el encuentro, es decir, el ensamble entre los dos modos de ser -el indio y el europeo-, lo que dio origen a una nueva cultura: la mestiza. Esta mezcla, supuso un ocultamiento de las divinidades masculinas y del Dios-Padre, extendiéndose, en cambio, una figura sincrética de la Virgen María, fusionada con las divinidades femeninas nativas ${ }^{25}$. La autora, sostiene que habría dos caminos posibles para explicar lo que pudo haber pasado en esta conjunción cultural. O

\footnotetext{
22 Consejo Episcopal Latinoamericano CELAM. Discípulos y Misioneros de Jesucristo para que nuestros pueblos en El tengan vida. Documento Conclusivo. CELAM; Aparecida, 2007.

23 Cf. S. MONTECINO, "Símbolo Mariano y constitución de la Identidad femenina en Chile" en Estudio Públicos. 39 (1990), pp. 283-290; S. MONTECINO. Madres y Huachos. Alegorías del mestizaje chileno. Editorial Sudamérica: Santiago, 1994; S. MONTECINO, "La huacha como construcción simbólica del género femenino o los indicios de una escena antropológica latinoamericana" en Stromata. 64/I-2 (2008), pp. 53-63.

24 Cf. S. MONTECINO, "Símbolo Mariano y constitución...”, p. 283.

25 S. MONTECINO, "Símbolo Mariano y constitución...”, p. 285.
} 
bien lo europeo y lo nativo se encontraron a través del símbolo universal de la "madre tierra" como eje de todo lo creado, o "lo mestizo", al quedar relegado a la soledad, se aferra a la imagen de la Madre-diosa como símbolo de cobijo y fortaleza. Esto último se entiende bajo el concepto de "huacho", propio de los estudios de Montecino. El término, hace referencia a los hijos vástagos que nacieron fruto de la unión entre el español y la mujer indígena, cuya familia tenía como eje la figura de la madre. Mientras la figura paterna es lejana y ausente -aunque no por ello carente de poder- la figura materna es la preponderante y la que domina el ethos familiar. En esta lógica, aparece la figura de la Virgen María como la divinización de la madre y quien define la identidad de las mujeres. Así, la madre celestial y las madres terrenas asumen la protección, subsistencia y afecto de sus hijos-huachos ${ }^{26}$.

En síntesis, lo que Montecino plantea es que muchos de los rasgos que están contenidos en el modelo mariano maternal, son visibles en la identidad de las mujeres chilenas. Esto significa que el horizonte religioso y simbólico tiene todavía un importante papel en la construcción de las identidades de las mujeres, cuestión que, bajo mi punto de vista, da relevancia a la reflexión marialógica. Frente a esto, considero que el desafío será cómo pensar una teología de María que se haga cargo de nuestra historia y del modo en cómo se gestó nuestra cultura, sin, al mismo tiempo, representar un obstáculo para las búsquedas liberadoras de las mujeres.

La teóloga Elina Vuola, si bien no es latinoamericana, ha desarrollado estudios de marialogía en relación con este contexto, por el cual se interesa especialmente. En uno de sus estudios, la teóloga finlandesa hace referencia ${ }^{27}$ a Evelyn Stevens, quien en 1973 proponía el término "marianismo" como uno de los rasgos distintivos de América Latina. Este concepto sirve para explicar el culto secular a la feminidad fundamentado en la devoción a la Virgen María. El marianismo hace de las mujeres sujetos semi-divinos, moralmente superiores y espiritualmente más fuertes que los varones. El punto es que el marianismo opera como la otra cara del machismo y, por tanto, permite que el ensalzamiento conviva con la opresión real que las mujeres sufren por parte de los varones ${ }^{28}$. Vuola retoma este término ya que le parece que permite comprender la distancia que existe entre el elevado papel

26 S. MONTECINO, "Símbolo Mariano y constitución...", p. 288.

27 E. VUOLA, "María, mujer en la política. Nuevos desafíos para la teología latinoamericana" en Albertus Magnus. 4/2 (2012), pp.59-7I. Disponible en: https://doi.org/IO.I5332/s20II-977I.2012.0004.04. 28 Cf. E. VUOLA, "María, mujer en la política ...", p. 63. 
que se le otorga a María y el lugar que efectivamente las mujeres tienen en la sociedad y en la Iglesia. La relación entre la exaltación de María, de parte de las iglesias, y el estatus que las mujeres tienen en estas es directamente proporcional. A más marianas las iglesias, más excluidas las mujeres ${ }^{29}$.

El marianismo está en relación con lo planteado por Montecino respecto a la estructura familiar de la sociedad chilena. En muchas de las familias chilenas, todavía hoy, la figura paterna o es débil o derechamente está ausente, sobre todo en contextos vulnerables. La madre aparece como la figura acogedora y protectora de sus hijos -incluso también de la pareja, quien es tratado como un hijo más ${ }^{30}-$ la que, generalmente, se combina con una identidad de macho, que exige un comportamiento sexual agresivo e, incluso, violento del varón. En la cultura chilena el marianismo no ha sido destituido ni reemplazado completamente, por tanto, la identificación de las mujeres con la madre sigue funcionando en la construcción de sus identidades ${ }^{31}$. Así, la maternidad se vuelve ambigua, pues, por un lado, es un elemento diferenciador y, por otro, tal y como es entendida y vivida, pareciera más un obstáculo que un vector para la realización plena de las mujeres.

Comprender el proceso histórico que ha ligado fuertemente a las mujeres a un concepto de maternidad, cuyo símbolo divino es la Virgen María, pone de manifiesto que esta concepción está mediada por la cultura y por el modo en cómo se comprenden y se viven las relaciones sociales entre los seres humanos ${ }^{32}$. Esto supone, por lo tanto, la posibilidad de interrogar dicha comprensión y abrir nuevos caminos, que permitan a las mujeres "escapar en busca de aire fresco" 33 . Me parece que este es justamente uno de los aportes de las marialogías feministas a la cultura y religiosidad chilena. Preguntarse y poner en cuestión la aparente inseparabilidad del binomio mujeres-maternidad y, por otro, maternidad-María, como el modelo de mujer-madre que todas las demás deben seguir y aspirar a cumplir.

La relación maternidad-María conecta con el dogma mariano fundamental que la Iglesia definió en Éfeso el año 43I impulsado, en parte, por las disputas cristológicas en torno a la unidad de la naturaleza

\footnotetext{
29 E. VUOLA, "María, mujer en la política ...", p. 67.

30 Cf. S. MONTECINO, "Símbolo Mariano y constitución...”, p. 289.

3I Cf. S. MONTECINO, "La huacha como construcción simbólica...”, p. 62.

32 Cf. S. MONTECINO, "La huacha como construcción simbólica...”, p. 54.

33 E. A. JOHNSON. Verdadera hermana nuestra..., p. 91.
} 
humana y divina de Cristo. Pero también por el sensus fidelium que proclamaba y creía en la maternidad divina de María: la Theotokos. María es la madre de Cristo, pero también la madre de las y los creyentes. Así, la relación que tenemos con ella es, sobre todo, una relación filial. Este dogma fundamental de la tradición se conjuga en nuestro contexto, como ya se ha expuesto, con una cultura que sobrevalora la maternidad. En esta línea, Azcuy rescata la reflexión de González Dorado quien plantea que es posible describir la cultura latinoamericana mediante el binomio machismo-maternidad ${ }^{34}$. Desde Montecino, este binomio también sería aplicable al contexto chileno lo que, bajo mi punto de vista, nos da una aproximación al modo en cómo se comprende y vive la maternidad en Chile. Porque el punto no es negar ni minimizar el dato de que María es la madre de Jesús, sino que la pregunta de fondo es de qué maternidad se trata, cómo entendemos ese concepto y cómo lo vivimos de manera de no caer en estereotipos de género que sean perjudiciales para las mujeres.

El modelo de maternidad, funcional a una pauta androcéntrica, que presenta a María como una mujer abnegada, obediente y que sabe sufrir en silencio por el fracaso y dolores de su hijo ${ }^{35}$, parece no estar muy lejos de la manera en cómo las mujeres chilenas viven su maternidad: en la soledad de criar y sostener a sus "huachos", con padres ausentes $\neg$-aunque autoritarios y dominantes-y, por tanto, sabiendo que tendrán que luchar solas por sacar a sus hijos adelante. Al mismo tiempo, esta concepción de la maternidad confluye con el hecho de que la maternidad es fuente de orgullo y sentido, pues, dada la valoración que esta tiene en la cultura, es el modo en cómo las mujeres se sienten reconocidas y el camino por el cual pueden alcanzar la plenitud de su ser mujer ${ }^{36}$.

Frente a este concepto de maternidad con sesgo androcéntrico y que presenta un ideal opresivo y restrictivo de la experiencia de ser madre -al mismo tiempo que excluye y discrimina a todas las mujeres que no lo son-, me parece que las marialogías feministas pueden ser un aporte. Por un lado, abriendo camino para acercarnos a la maternidad divina de María de un modo nuevo, es decir, no idealizado ni construido exclusivamente desde los clérigos (varones y célibes). Y, en conexión con

\footnotetext{
34 Cf. V. R. AZCUY, "María en diversas voces de la teología latinoamericana. Algunos ciclos de una interminable conversación en las últimas tres décadas" en Ephemerides Mariologicae. 62/4 (2012), pp. 373-402, 386-389.

35 Cf. D. IRARRÁZAVAL, "María en el cristianismo latinoamericano" en Concilium. $327 / 4$ (2008), pp. IO7-II6, p. II2. Disponible en: https://www.revistaconcilium.com/wp-content/uploads/20I9/ pdf/327.pdf.

36 Cf. M. P. SILVEIRA, "María en la teología: ¿reflexiones elaboradas en despachos o en fronteras?" en ATeo. 2I/57 (2017), pp. 495-516, p. 306.
} 
esto, al resignificar la maternidad de María, repensar, interpretar y vivir la maternidad de las mujeres chilenas de un modo liberador. Al mismo tiempo, esto impactaría en la comprensión y modo en cómo se vive la paternidad. Pues esta nueva comprensión de las mujeres y la maternidad abriría el camino para que también los varones se entiendan a sí mismos y a la paternidad de un modo nuevo. En este sentido, compartimos la pregunta que levanta el teólogo chileno Diego Irarrázaval ¿qué es lo que la marialogía aporta? ${ }^{37}$

Ahora bien, según mi modo de ver, esta reflexión acerca del estrecho vínculo entre marianismo y culto a María -como la mujer/ madre ideal-, debiera tener en cuenta el hecho que, a pesar de la opresión $\mathrm{y}$ androcentrismo que conlleva, al menos en el caso chileno, no implica un detrimento de la devoción a la Virgen. Al contrario, al día de hoy, uno de los rasgos propios del país sigue siendo su veneración mariana. Lo especialmente curioso es que, si bien la devoción mariana también se da en los varones, la gran mayoría de las personas que acuden a los santuarios, realizan mandas, celebran el mes de María, rezan el rosario, entre otras actividades de la misma índole, son mujeres. Una posible explicación es que las chilenas, o no se sienten oprimidas por este modelo, o es algo que simplemente aceptan como parte del estereotipo que les corresponde encarnar. Pero, otra respuesta posible es que ven en María algo más que un modelo de maternidad ideal. Si es así, ¿qué es lo que ven en ella que las hace acudir a su persona en busca de aliento y consuelo, de fuerza y esperanza en sus penosas luchas de cada día por sobrevivir ellas y sus hijas(os)?

Clara Temporelli, en su estudio sobre la religiosidad popular mariana, da una pista interesante. Ella plantea que, por un lado, las mujeres se identifican con María porque ella ha compartido el sufrimiento, la tragedia humana ${ }^{38}$. En la misma línea, está lo que plantea Carmiña Navia, quien propone que la cercanía se produce porque María entiende sus sufrimientos debido a que ella también los padeció. «Saben que parió con dolor en un pesebre porque fue rechazada en la posada, saben que una espada atravesó su corazón porque ajusticiaron a su hijo. Sienten que esa figura que las antecedió en la pena las ilumina y las consuela» ${ }^{39}$.

37 Cf. D. IRARRÁZAVAL, "María en el cristianismo...", p. I07.

38 Cf. M. C. TEMPORELLI, María a la luz de la fe del pueblo latinoamericano. Nuestra Señora de Guadalupe. GRAM: Buenos Aires, 2013, p. 37.

39 C. N. VELASCO, "María, la mujer (¿la diosa?) que consuela" en Albertus Magnus. 4/2 (2012), pp. 4I-57, p. 56. Disponible en: http://dx.doi.org/I0.I5332/s20II-977I.20I2.0004.03. 
Este tema de la connaturalidad que el pueblo tiene con $\mathrm{Marí}^{40}$ no puede darse por sentado. El mismo documento de Aparecida reconoce el valor y la importancia de la religiosidad popular como un lugar de encuentro con Jesús y la trascendencia ${ }^{4}$. En este sentido, creo que la propuesta de una marialogía feminista para nuestro contexto no puede sino considerar la religiosidad popular de nuestro pueblo, como una de las características propias del mismo, a pesar de que ello suponga algunos desafíos y dificultades.

\section{Desafíos desde el contexto chileno para una marialogía feminista}

Si lo que intentamos en este estudio es, en diálogo con Johnson, dilucidar cuáles podrían ser sus aportes para una reflexión sobre María pensada desde el contexto chileno, descubrimos que el soporte antropológico de su propuesta permite plantear una marialogía que resulte liberadora. Ahora bien, al confrontarnos con la cultura chilena y sus rasgos propios, nos dimos cuenta de que el binomio María-maternidad está asociado al modo en cómo se construye la identidad de las mujeres chilenas y que, además, es la contraparte del machismo. Por tanto, se reconoció que uno de los aportes que la teología feminista de María puede hacer, está justamente en el modo en cómo se avanza hacia una comprensión no sexista de la maternidad de María y, al mismo tiempo, se disocia el binomio maternidad-mujeres, como si ésta fuera la única manera en que ellas pueden alcanzar la realización y plenitud de sus vidas. Por otra parte, se veía la necesidad de que esta reflexión no sea ciega a un dato fundamental de nuestro contexto: el hecho de que, sobre todo las mujeres, siguen acudiendo a María, no viendo necesariamente en ella un modelo que las limite, sino una fuente de consuelo y fortaleza para su vida diaria. ¿Qué desafíos entonces levanta la religiosidad popular a una marialogía pensada desde nuestro contexto?

Adentrarnos, aunque sea de forma introductoria en esta cuestión, me parece relevante. Porque si bien es clara la necesidad de repensar el modelo mariano para que este no sea un obstáculo sino fuente de inspiración y esperanza para las mujeres, creo que el modo en cómo llevamos adelante la reflexión resulta no menos importante y habrá que evitar dos cosas. Por un lado, no caer-aunque con la mejor de las intenciones- en la imposición de un modelo, por muy liberador para las mujeres que nos parezca. Al contrario, este tiene que ser respetuoso de la historia y tradición devocional del pueblo, siendo capaz de recoger lo

40 M. C. TEMPORELLI, María a la luz de la fe..., p. 36.

4I Cf. CELAM. Discípulos y misioneros..., n²63, p.I5I. 
mejor de ella. Ahora bien, esto no significa desconocer la importancia de revisar y cuestionar cómo se está entendiendo la figura de María y de qué tipo de maternidad se trata. Por tanto, el desafío y la tensión están -en palabras de Diego Irarrázaval (2008)-, por una parte, en confrontar el marianismo porque desfigura a María y perjudica a las mujeres y, por otra parte, apreciar el culto mariano con sus vetas humanizadoras ${ }^{42}$.

Si bien la cuestión de la religiosidad popular es un tema que se encuentra prácticamente ausente en la teología de María de Johnson -crítica que comparto con María Josefina Llach en su recensión sobre la obra-, me parece que una marialogía feminista pensada desde Latinoamérica, no puede sino acercarse y hacerse cargo del modo particular cómo el pueblo vive y manifiesta su devoción a la madre de Jesús. La obra colectiva Vírgenes y diosas de América Latina ${ }^{43}$ es un buen ejemplo del esfuerzo por comprender cómo la religiosidad popular mariana reúne y condensa las creencias de los pueblos originarios previas a la conquista y cómo esto puede reconocerse hoy a través de las fiestas religiosas marianas.

Por otra parte, esta es también la intuición de Vuola en su obra The Virgin Mary across Cultures. Devotion among Costa Rican Catholic and Finnish Orthodox Women ${ }^{44}$. En ella, la teóloga finlandesa se hace cargo de la tensión entre la concepción negativa que la marialogía feminista tiene del modelo mariano y el hecho concreto que las mujeres acudan a María. Es por esto, que Vuola subraya la necesidad de que preguntarles a las mismas mujeres quién es María para ellas ${ }^{45}$. Para la teóloga, el punto está en que esta pregunta no puede contestarse solamente desde la teología o la doctrina, sino que la respuesta debe incluir y considerar las experiencias de las mujeres ${ }^{46}$. Para esto, plantea la importancia de incluir métodos de investigación que puedan dar cuenta de sus vivencias, creencias y comprensiones, utilizando, en su caso, métodos etnográficos.

Además del trabajo que Vuola hace comparando las experiencias de mujeres finlandesas y mujeres de Costa Rica, también se puede citar la tesis doctoral de María Pilar Silveira que trata sobre las expresiones

42 Cf. D. IRARRÁZAVAL, "María en el cristianismo...", p. II3.

43 V. CORDERO et al. (coords.), Vírgenes y Diosas en América Latina: la resignificación de lo sagrado. Doble Clic: Montevideo, 2004. Disponible en: http://conspirando.cl/wp-content/uploads/20I6/05/ V\%C3\%ADrgenes-y-Diosas-de-Am\%C3\%Agrica-Latina.pdf.

44 E. VUOLA. The Virgin Mary across Cultures. Devotion among Costa Rican Catholic and Finnish Orthodox Women. Routledge: New York, 2019.

45 Cf. E. VUOLA., The Virgin Mary across Cultures..., p. 2.

46 Cf. E. VUOLA., The Virgin Mary across Cultures..., pp. 5-6. 
populares de devoción en las advocaciones marianas venezolanas. Por medio de la realización de encuestas, el estudio de cuadernos de intenciones y observaciones ${ }^{47}$, la autora ofrece un amplio y rico panorama de la religiosidad vivida en Venezuela. Es interesante, en general, que Silveira reconoce una vivencia positiva de la religiosidad mariana de las mujeres, quienes acuden a María como alguien cercano y con quien se identifican, al mismo tiempo que es capaz de ayudarlas e inspirarles esperanza y fortaleza.

A mi juicio, hay aquí una diferencia importante entre este modo de pensar el tratado y la manera en cómo Johnson lo desarrolla en su obra. Si bien, como ya se ha señalado, la teóloga norteamericana intenta dar cuenta del vasto panorama marialógico en las distintas latitudes del mundo, este esfuerzo "universalista" impide que se consideren las experiencias individuales y concretas de las mujeres. En esta línea, y sin desconocer el valor y el amplio y bien fundamentado trabajo que Johnson lleva adelante, creo que, para una futura investigación, se podría incursionar en la pregunta acerca de cómo dar voz a las mujeres chilenas del pueblo respecto de las experiencias de su devoción a María. Porque, desde mi punto de vista, solamente escuchando las voces de las mujeres, se podrá construir una teología feminista que respete las interpretaciones y realidades de la religiosidad popular. En el fondo, la pregunta sería cómo acercarse a la religiosidad vivida de las mujeres. Para este trabajo, será importante tener en cuenta la advertencia de Temporelli: "el pueblo no parte de conceptualizaciones sino de situaciones; no hace disquisiciones, sino que vive; no define, sino que siente" ${ }^{48}$. Al mismo tiempo, esto refuerza la intuición de Vuola expuesta más arriba, sobre la necesidad que el pensamiento teológico feminista y las experiencias y vivencias de las mujeres no corran por carriles paralelos sino que, dialoguen y se enriquezcan mutuamente ${ }^{49}$.

En este sentido, considero que sería enriquecedor complementar el vasto panorama que Johnson hace en su obra de la cultura antigua en la que Miriam de Nazaret se desenvolvió, con un análisis de la cultura chilena actual, para acercarse a la comprensión de la figura de María y la vivencia de la devoción hacia ella en el presente. Bajo mi punto de vista, esto permitiría hacerse cargo de un segundo desafío que nuestro contexto presenta para la teología de María de Johnson y que hemos

47 M. P. SILVEIRA. Mariología popular latinoamericana. Fisonomía de la Mariología popular venezolana, Arquidiócesis de Mérida: Caracas, 20I3, p. 26.

48 M. C. TEMPORELLI, María a la luz de la fe..., p. 29.

49 Cf. E. VUOLA., The Virgin Mary across Cultures..., p. 26. 
presentado en el apartado anterior: la cuestión de la maternidad. Si bien la teóloga norteamericana no niega la maternidad de María como una de las facetas importantes de su identidad y figura, a lo largo de la obra pone el acento, más bien, en el sustantivo de hermana. ¿Cómo se recepciona esto en un país como Chile donde, como ya se explicó, la maternidad tiene un rol central en la cultura? De este hecho, dan cuenta Gebara y Bingemer: «para la mayoría de la población, incluso en las CEBs, la madre del cielo, santa y misericordiosa, parece prevalecer aún sobre la hermana de la tierra ${ }^{50}$. ¿Ha cambiado esta percepción en las últimas décadas en la sociedad chilena?

Me parece que un estudio empírico con trabajo de campo como los que mencionamos más arriba- que pueda dar cuenta de la experiencia vivida de las mujeres podría darnos luces para esta pregunta y así enriquecer enormemente las propuestas marialógicas en sus concreciones de acuerdo con cada uno de los contextos. Porque, al menos desde mi experiencia, en Chile sigue siendo muy fuerte la sobrevaloración de la maternidad. En contextos de pobreza, la maternidad da a las mujeres un estatus que no tienen las demás. Como ya se expuso, Montecino afirma que la maternidad es un elemento diferenciador y que, a la vez, permite a las mujeres alcanzar la plenitud de su ser femenino. Entonces, ¿quién no quisiera ser madre para ser -por lo menos algo más- vista, oída, reconocida? Cuando se siente que no se tiene nada, la maternidad abre la posibilidad de tener, al menos, un(a) hijo(a) propio.

Entonces, dado que en el contexto latinoamericano la maternidad no puede ser una faceta subestimada, volvemos a nuestra pregunta: ¿cómo pensamos y proponemos una teología feminista que dé cuenta de María como una mujer que vivió su maternidad de una manera liberada y liberadora? ¿Sería posible afirmar algo así? ¿Cómo convergerían en María la maternidad junto con la sororidad (hermandad) y el discipulado? ¿Es posible esta conjunción o son elementos que entran en tensión? Me parece que el trabajo bíblico que Johnson desarrolla en la quinta parte de su obra, "Un mosaico: la memoria peligrosa de María", ofrece una valiosa fundamentación bíblica para descubrir, en palabras de Mercedes Navarro, que María tiene muchos rostros ${ }^{51}$.

En síntesis, lo que se ha intentado mostrar en este estudio es la

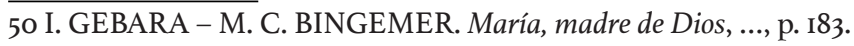

5I Cf. M. NAVARRO. Los rostros bíblicos de María. Exégesis y hermenéutica bíblica feminista. Verbo Divino: Navarra, 2020. 
importancia de considerar la realidad sociocultural de las mujeres y sus diferentes contextos como una rica veta que las marialogías feministas pueden seguir trabajando en el futuro. Si bien hay algunos núcleos o elementos comunes, otros muchos guardarán relación con la realidad de las mujeres de cada tiempo y lugar. En el caso chileno, la consideración de la forma en que se entiende y vive la maternidad, por un lado, y la religiosidad popular, por otro, son dos elementos que no pueden quedar fuera de la reflexión.

Me parece que es justamente la consideración de estos elementos particulares de cada contexto lo que permite hacerse cargo de la diversidad y pluralidad propias del género humano, y, por tanto, pensar y proponer una marialogía que sea realmente inclusiva. 